\title{
Advanced systems in public transport, with a touch of data
}

\author{
Martin Trépanier ${ }^{1} \cdot$ Mark Hickman $^{2}$
}

Accepted: 1 November 2021 / Published online: 8 November 2021

(c) The Author(s), under exclusive licence to Springer-Verlag GmbH Germany, part of Springer Nature 2021

This special issue of Public Transport: Planning and Operations assembles selected papers from the 14th International Conference on Advanced Systems in Public Transport (CASPT), that was held in conjunction with the 4th International Workshop and Symposium on Research and Applications on the Use of Passive Data from Public Transport (TRANSIT DATA) in Brisbane, Australia, in July 2018.

The conference was the first occasion to blend research work from the CASPT community to the one from researchers that directly use passive data, like smart card fare collection system data, to propose new ways to address the advanced planning of public transport systems. As a result, this issue presents a wide spectrum of papers that covers various topics and methods.

Aboudina et al. propose a macroscoping approach to evaluate bus bridging scenarios for railway disruption management. The case study of Toronto, Canada, is used to illustrate the method.

Arbex et al. evaluate the improvements made to a bus network by comparing the before-and-after performance indicators. Their work shows the use of smart card data to assess changes in the demand side, and they use an accessibility analysis for the supply side.

Berggren et al. show that the pre-trip information provided to passengers has a significant influence on waiting times. They use a smart phone application to conduct a survey and combine its results with GTFS and AVL data. A statistical analysis is then used to create and characterise archetypes of users that show different explanatory factors in the effect of pre-trip information.

Degeler et al. used a clustering method to analyse the phenomenon of vehicle bunching in public transport networks. They extract isolated bunching swing formations and propose a classification of these events, looking at their characteristics.

Martin Trépanier

mtrepanier@polymtl.ca

Mark Hickman

m.hickman1@uq.edu.au

1 CIRRELT and Polytechnique Montreal, Université de Montréal, CP 6128, Succursale Centre-Ville, Montréal, QC H3C 3J7, Canada

2 Faculty of Engineering, Architecture and Information Technology Brisbane St Lucia, School of Civil Engineering, Brisbane City, QLD 4072, Australia 
Fournier et al. present an $\mathrm{A}^{*}$-guided algorithm with Pareto dominance-based elimination to address the multi-objective bus passenger trip planning problem. They test their approach in two Brazilian cities to help improve the results of the trip-planning tool.

He et al. propose a clustering method, based on smart card data, to regroup public transport passengers accordingly to their space-time trip profile. They use dynamic time warping distances that combine the points in time of one day and their corresponding locations where smart card transactions are made.

Ljunggren et al. develop a maximum bottleneck path algorithm to insert an additional train path in an existing railway timetable, while keeping its robustness and minimising the effects on passengers.

Matos et al. combine the Boolean satisfiability problem (SAT) and reinforcement learning approaches to solve periodic timetabling problems. The addition of machine learning helps to provide good results in comparison to exact approaches.

van Mil et al. characterise the factors affecting the combined bicycle-transit mode. An approach that combines a literature review with a stated-preference survey is used to identify the factors that bring travellers to use a bicycle to access public transport.

Sessa et al. present a hybrid stochastic approach to reconstruct the trajectories of trains in the case of missing or unreliable data. They test their method on a Swiss line, integrating GPS data and power measurements at traction units.

Publisher's Note Springer Nature remains neutral with regard to jurisdictional claims in published maps and institutional affiliations. 